\title{
Computed Tomography of Primitive Neuroectodermal Tumors
}

\author{
S. Ramaiah Ganti, ${ }^{1}$ A. John Silver, ${ }^{1}$ Paul Diefenbach, ${ }^{2}$ Sadek K. Hilal, ${ }^{1}$ Michel E. Mawad, ${ }^{1}$ and Paul Sane ${ }^{1}$
}

The primitive neuroectodermal tumor is a neoplasm of young individuals that occurs predominantly in the supratentorial compartment. It appears on computed tomography as a bulky mass, often with calcification with predominantly homogeneous enhancement but on occasion with cystic or necrotic areas. Cerebrospinal fluid seeding was observed in more than half the cases in our series. The patients had a short survival period (7-24 months). Despite surgery, radiation, and chemotherapy, further growth of this tumor has not been prevented.

In 1973, Hart and Earle [1] described a group of brain tumors with common pathologic and clinical features, which they termed "primitive neuroectodermal tumors." These are usually found in the cerebrum of children and young adults. They are undifferentiated and show mixed glial and neuronal elements. These tumors may have a variety of names: primary cerebral neuroblastoma, cerebral medulloblastoma, unclassified glioma, or undifferentiated small-cell neoplasm [1].

This report reviews the computed tomographic (CT) findings of 13 histologically proven primitive neuroectodermal tumors scanned between 1974 and 1982. Our goal was to be able to make a reasonably certain preoperative diagnosis with $\mathrm{CT}$, so that the clinician can know the tumor behavior and its rapid course and, hence, proper and more aggressive therapy may be planned.

\section{Materials and Methods}

From 1960 to 1982,22 cases of primitive neuroectodermal tumors were diagnosed at the Neurological Institute of New York. CT scans were obtained before and after contrast injection in 13 patients. There were eight males and five females in the group. All but one of the patients were below the age of 16 years. Clinically, the patients presented with signs of increased intracranial pressure, evident by headache and papilledema, as well as hemiparesis and focal seizures. One patient in whom the primary tumor was in the posterior fossa presented with spinal cord symptoms. Cerebral angiograms were obtained in six patients. Three patients had subsequent myelograms for spinal cord and/or nerve root symptoms.

\section{Results}

Among 13 patients, CT revealed gross calcification in six. The calcifications were irregular, coarse, nodular, and located in the central and peripheral parts of the tumor. In addition, these six cases had hypo- and hyperdense areas in the tumor, when compared with the surrounding brain, but no cerebrospinal fluid (CSF) densities. Two cases were hypodense, three were isodense, one case had a hyperdense area, and one case presented with hemorrhage. Even before contrast injection, the tumor margins were relatively sharp.

All cases showed contrast enhancement. Eight cases revealed tumors that densely enhanced in a homogeneous fashion. This pattern was termed "solid" enhancement. Five cases revealed a "ring" enhancement with areas of hypodensity which might represent a necrosis or cyst. The solidly enhancing group often had a large margin abutting the meningeal surface and passing from one intracranial compartment to another, for example, supratentorial to infratentorial or one hemisphere to the other.

In all but one case, the tumor was located in the supratentorial compartment. In five cases, the tumor was located in the frontal lobes, in four cases in the temporal lobes, of which two were located in the medial temporal lobe with encroachment on the suprasellar cistern. In two cases, the tumor was in the parietal lobe and in one case it was in a deep location involving the insula. One tumor was in the posterior fossa. The posterior fossa tumor was outside the fourth ventricule, in the region of the foramen magnum, in the vallecula, so it was not a typical medulloblastoma. In all cases, the tumor was bulky with sharp boundaries and little surrounding edema. Bony changes occurred in only one case, although the mass was usually close to the meningeal surface and the inner table of the calvaria. CSF seeding was noted intracranially in nine cases as evident by the prominent enhancement of the adjacent subarachnoid space and/or ventricle. Cerebral angiography, in three of six cases, demonstrated neovascularity in the internal carotid circulation, associated with a tumor "blush" and contrast accumulation within cystic or necrotic areas during the late phase of angiography. No early veins were detected. One of these three patients had a delayed CT scan showing contrast in a large cystic cavity (fig. 1). Myelography was performed in three patients, and tumor seeding was noted with multiple intradural extramedullary defects in all three cases (fig. 2).

\section{Discussion}

Grossly, primitive neuroectodermal tumors or primary cerebral neuroblastomas are well defined, sharply demarcated tumors with a lobulated, soft, or granular pearly gray cut surface. Foci of cystic degeneration are often present. Histologically, the tumor is highly cellular with predominantly small undifferentiated cells. These have dark oval or irregular nuclei without observable cystoplasm [1].

\footnotetext{
'Department of Radiology, Neurological Institute, Columbia-Presbyterian Medical Center, 710 W. 168th St., New York, NY 10032. Address reprint requests to S. R. Ganti.

${ }^{2}$ Department of Neurosurgery, Neurological Institute, Columbia-Presbyterian Medical Center, New York, NY 10032.

AJNR 4:819-821, May/June 1983 0195-6108/83/0403-0819 \$00.00 (c) American Roentgen Ray Society
} 

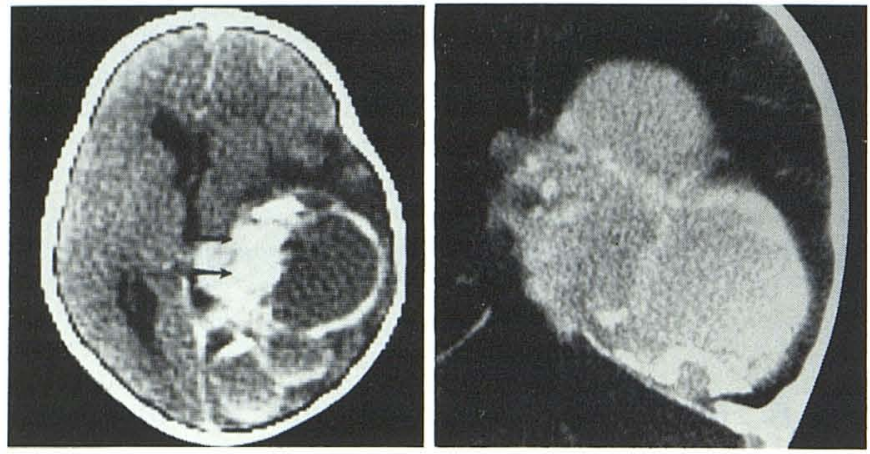

A

B

Fig. 1.-Ring-enhancing primitive neuroectodermal tumo: A, After contrast. Multiple ring-enhancing tumor with lucent areas and calcifications (arrow). B, Delayed scan, coronal view, after contrast. Lucent areas filled with contrast material. Intraventricular extension of enhancing tumor, which crosses midline.

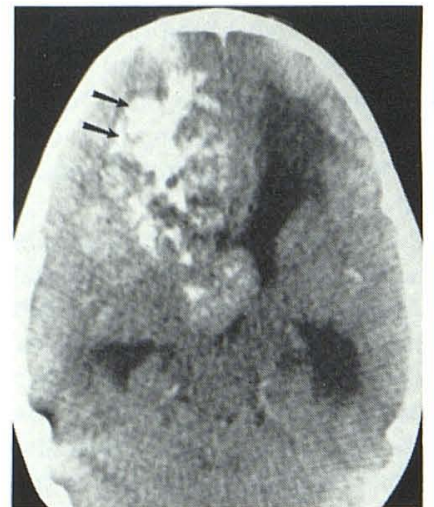

A

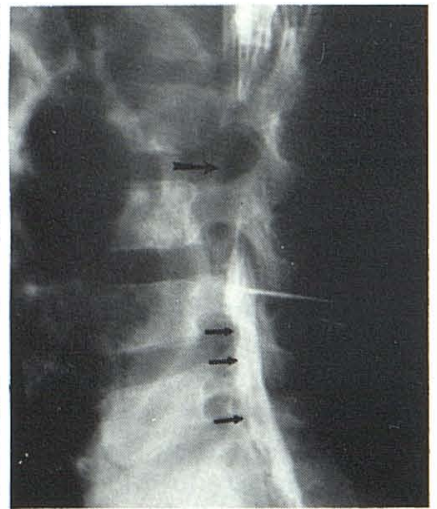

B
Fig. 2.-Primitive neuroectodermal tumor (5-year-old boy). A, After contrast. Irregular calcifications (arrows) with enhancing component in right frontal lobe extending into septum pellucidum and third ventricle. B, Lateral view of metrizamide myelogram. Incomplete extradural block (large arrow) with multiple intradural defects (small arrows) on ventral aspect of thecal sac, consistent with tumor seeding.
Three pathologic features distinguish primitive neuroectodermal tumors from other central nervous system tumors. First, unlike most malignant gliomas, they usually show an abrupt transition between the tumor and adjacent brain; occasionally there is microscopic infiltration. Second, hypertrophic neuroglial reaction may be present along the edge of the neoplasm; this pattern resembles that seen in invasive sarcomas. Third, they have a fibrous connective tissue stroma [2].

Neuroectoderm is a term that has been used to describe the total substance of the central nervous system excluding the mesenchymal elements, which are blood vessels and microglia [1]. Primitive neuroectodermal or neuroepithelial are the terms used to describe tumors composed of undifferentiated cells resembling germinal matrix [3]. These terms have been used to describe various tumors, most often those that arise in the cerebellum of children and that have traditionally been called medulloblastomas [4]. The cerebral examples of this type have more often been called cerebral neuroblastomas. The latter term, however, is too restrictive in view of the variable yet frequent glial, as well as neuronal, differentiation of the tumors.

Primitive neuroectodermal tumors, as defined by Hart and Earle [1], more accurately expresses the characteristics of this group with glial and neuronal elements. The other names used in the literature for primitive neuroectodermal tumors are cerebral neuroblastoma, cerebral medulloblastoma, undifferentiated small-cell neoplasm, and unclassified glioma.

There are at least two reports describing the CT features of these primitive neuroectodermal tumors under the name of primary cerebral neuroblastoma $[5,6]$. The initial series reported by Chambers et al. [5] included 11 patients, of whom nine had CT studies. The CT findings included gross calcification and areas of hypodensity, isodensity, and mixed density; there was variable enhancement after contrast administration. In a series of 11 cases of intracranial neuroblastoma reported by Zimmerman and Bilaniuk [6] there were three primary cerebral neuroblastomas. Two had a high-density area which was interpreted as an intratumoral hemorrhage, and the other presented as an intraventricular mass.

On CT scans from our study of 13 cases, these tumors are large and often demonstrate gross calcification associated with either solid or ring enhancement, the latter associated with cystic or necrotic areas. The ring enhancement of some of the cystic or necrotic tumors may resemble that of an abscess, and a resemblance is also seen in gross pathologic specimens (fig. 3). For reasons that are unclear, these tumors show no significant associ-
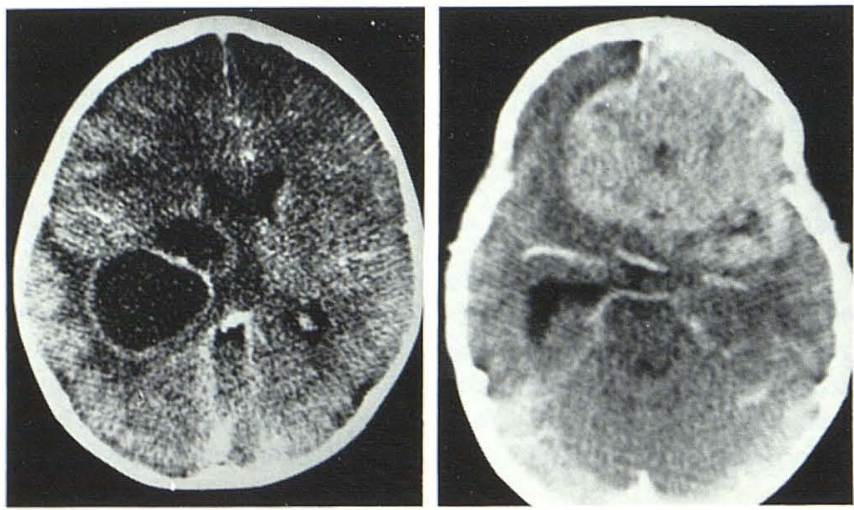

3

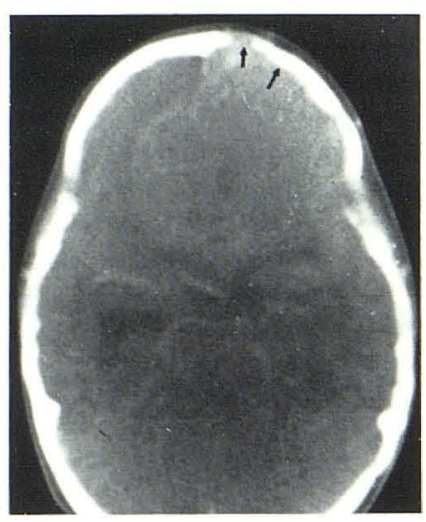

4B
Fig. 3.-Ring-enhancing primitive neuroectodermal tumor. CT scan after contrast reveals ring-enhancing lesions, which can simulate abscess.
Fig. 4.-Solidly-enhancing primitive neuroectodermal tumor (5-year-old girl). A, After contrast. Homogeneously enhancing tumor in left frontal lobe close to inner table. B, Same view at bone window setting. Erosion of calvarium (arrows). 

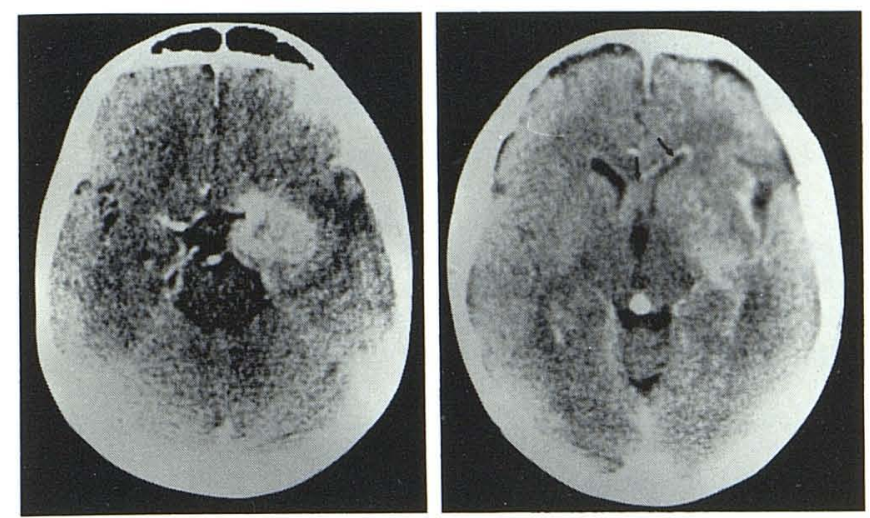

A B

Fig. 5.-Parasellar primitive neuroectodermal tumor (the only adult patient, a 55-year-old man). A, After contrast. Enhancing mass in parasellar area encroaching on suprasellar cistern; this appearance can mimic meningioma. B, Higher level. Septal and periventricular enhancement (arrows) suggest primitive neuroectodermal tumor with seeding into CSF spaces, rather than meningioma.

ated edema despite their large size. The apparent sharp demarcation of their edges from brain tissue and the presence of central necrosis often suggest a diagnosis of metastatic neoplasm. Parenchymal central nervous system metastases, however, are rare in young children [7]. Although these tumors are very large and close to the inner table of the calvarium, bone changes were noted in only one case (fig. 4), probably because these large tumors grow too rapidly to produce bone changes.

In the group of eight solidly enhancing tumors, there was a large component of the mass abutting the meningeal surface and the tumor frequently extended from one intracranial compartment to another. These features were rarely seen in other intraaxial tumors. In the two cases of medial temporal tumors with diffuse involvement of the suprasellar cistern, the tumor was difficult to differentiate from an extraaxial lesion. However, these medial temporal lobe lesions had seeding into the CSF spaces; this was more consistent with the diagnosis of a primitive neuroectodermal tumor than of an extraaxial lesion such as a meningioma (fig. 5). Since most of the tumors in our series enhanced in a dense and relatively homogeneous fashion with small nonenhancing areas, which on one delayed scan filled with contrast material, a delayed scan after contrast enhancement is recommended for suspected cases of primitive neuroectodermal tumor.
Primitive neuroectodermal tumors are known to metastasize via CSF pathways [3]. In our series, nine patients had CSF seeding, manifested on CT by enhancement of the subarachnoid space and the ventricular walls. One patient had spinal seeding initially, and two patients developed it subsequently.

Although CT findings of primitive neuroectodermal tumors are not specific, the constellation of a bulky tumor with sharp margins and dense and relatively homogeneous enhancement associated with cystic or necrotic areas and gross calcification usually suggests the diagnosis of primitive neuroectodermal tumor. In addition, the presence of CSF seeding may help in differentiating this tumor from other gliomas. Finally, the age group of children and young adults reduces the likelihood of metastatic disease.

Clinically, these patients have a relatively short duration of symptoms. The survival period is also short. A review of 54 cases of primitive neuroectodermal tumors reported by various authors reveals that the age of tumor onset is from birth to 24 years. The average survival period is $7-24$ months [8]. This is consistent with our series. The therapy plan as suggested by Duffner et al. [8] is surgical decompression of the tumor followed by radiation therapy and a chemotherapy protocol. The entire neuroaxis should be included in the irradiated field because of the tendency of these tumors to invade the leptomeninges.

\section{REFERENCES}

1. Hart MH, Earle KM. Primitive neuroectodermal tumors of the brain in children. Cancer 1973;32:890-896

2. Rubinstein LJ. Cytogenetics and differentiation of primitive central neuroepithelial tumors. J Neuropathol Exp Neurol 1972;31:7-36

3. Fujita S. The matrix cell and cytogenetics in the developing central nervous system. J Comp Neurol 1962;120:37-42

4. Chatty EM, Earle KM. Medulloblastoma. A report of 201 cases with emphasis on the relationship of histologic variants to survival. Cancer 1971;81:977-983

5. Chambers EF, Turski PA, Sobel D, Wara W, Newton TH. Radiologic characteristics of primary cerebral neuroblastomas. Radiology 1981;139:101-104

6. Zimmerman RA, Bilaniuk LT. CT of primary and secondary craniocerebral neuroblastoma. AJNR 1980;1:431-434, AJR 1980;135:1239-1242

7. Vannucci RC, Borten M. Cerebral metastatic disease in children. Neurology (NY) 1974;24:981-985

8. Duffner PK, Cohen ME, Heffner RR, Freeman AI. Primitive neuroectodermal tumors of children. An approach to therapy. J Neurosurg 1981;55:376-381 